\title{
Long-term evaluation of warm-air treatment effect on adaptation of silane- containing universal adhesives to lithium disilicate ceramic
}

\author{
PaPa KAYKHINE ${ }^{1}$, Antonin TICHY',2, Ahmed ABDOU ${ }^{1,3}$, Keiichi HOSAKA ${ }^{1}$, Richard M. FOXTON ${ }^{4}$, Yasunori SUMI ${ }^{5}$, \\ Masatoshi NAKAJIMA ${ }^{1}$ and Junji TAGAMI ${ }^{1}$ \\ ${ }^{1}$ Department of Cariology and Operative Dentistry, Graduate School of Medical and Dental Sciences, Tokyo Medical and Dental University, Tokyo, \\ Japan \\ ${ }^{2}$ Institute of Dental Medicine, First Faculty of Medicine of the Charles University and General University Hospital in Prague, Czech Republic \\ ${ }^{3}$ Biomaterials Department, Faculty of Dentistry, Modern University for Technology and Information, Cairo, Egypt \\ ${ }^{4}$ Division of Conservative Dentistry, King's College London Dental Institute at Guy's King's and St Thomas' Hospitals, King's College London, UK \\ ${ }^{5}$ Department for Advanced Dental Research, Center of Advanced Medicine for Dental and Oral Disease, National Center for Geriatrics and \\ Gerodontology, Obu, Aichi, Japan \\ Corresponding author, Masatoshi NAKAJIMA; E-mail: nakajima.ope@tmd.ac.jp
}

The effect of warm air-blowing on the sealing ability of two one-bottle and one two-bottle silane-containing universal adhesives (UAs) applied to lithium disilicate ceramics (LDS) was evaluated. Tapered cavities prefabricated in LDS blocks (IPS e.max CAD) were treated by the adhesives, air-blown either with normal air $\left(23 \pm 1^{\circ} \mathrm{C}\right)$ or warm air $\left(60 \pm 5^{\circ} \mathrm{C}\right)$, and filled with a flowable resin composite. Interfacial gap formation was observed using swept-source optical coherence tomography immediately after filling, after $24 \mathrm{~h}, 5,000$ and 10,000 thermal cycles, and an additional 1-year water storage. Specimens bonded using normal air-blown one-bottle UAs exhibited a significantly higher gap formation than the two-bottle UA $(p<0.001)$, and detached within $24 \mathrm{~h}$. Warm air-blowing significantly decreased the gap formation of one-bottle UAs $(p<0.001)$, but the specimens dislocated within $24 \mathrm{~h}$ or during thermocycling. The twobottle UA withstood all aging procedures and warm air-blowing significantly decreased its 1-year gap formation compared to normal air-blowing $(p=0.002)$.

Keywords: Lithium disilicate ceramic, Universal adhesive, Silane, Warm-air treatment, OCT

\section{INTRODUCTION}

All-ceramic restorative materials have become increasingly popular for aesthetic tooth restoration because of improvements in the strength of ceramic materials and in the adhesion between tooth substrates and ceramics. However, a fracture of the ceramic is a one of the most frequent causes of restoration failure ${ }^{1}$. Because a complete replacement of the restoration is technically demanding, time-consuming and expensive, the repair of fractured restorations with resin composite is a faster and more cost-effective option ${ }^{2}$.

When luting glass ceramic restorations to tooth substrates, hydrofluoric acid etching has been conventionally used to create a superficial microstructure on the surface and promote micromechanical interlocking with resin luting materials ${ }^{3)}$. However, this step is not applicable for the repair of ceramic restorations in the oral environment because the use of hydrofluoric acid is hazardous ${ }^{4}$. In the absence of micromechanical interlocking, a silane coupling agent ${ }^{5}$ ) is required to achieve a chemical interaction between the ceramic surface and methacrylate-based adhesives ${ }^{6}$. The most commonly used silane is 3-methacryloxyproyltrimeth oxysilane ( $\gamma$-MPTS), which is activated by hydrolysis to a corresponding silanol that can interact with glass substrates. Hydrogen bonds with the glass-surface hydroxyl groups are initially formed and these are later replaced by stable siloxane bonds ${ }^{7}$.
The hydrolysis of $\gamma$-MPTS is accelerated in acidic conditions $\mathrm{s}^{8}$; therefore, acidic monomers are utilized as catalysts in silane coupling agents. Recently, $\gamma$-MPTS has been incorporated in some one-bottle self-etch adhesives which enabled adhesion to glass ceramics. This can also simplify the bonding procedure in case of restoration repair where combined adherend surfaces with enamel, dentin, resin composite and glass ceramics are present ${ }^{9}$. However, several studies have reported low bond strengths of the tested one-bottle silanecontaining universal adhesives to glass ceramics ${ }^{10,11}$. This was attributed to a premature hydrolysis of $\gamma$-MPTS in the acidic conditions of one-bottle self-etch adhesives followed by a dehydration condensation of silanols into oligomers which can no longer bond to glass ${ }^{12}$. This mechanism was also confirmed using nuclear magnetic resonance $(\mathrm{NMR})^{11)}$, X-ray photoelectric spectroscopy (XPS) and Fourier transform infrared spectroscopy $(\text { FTIR })^{13)}$. A simple way to prevent the pre-hydrolysis and self-condensation is to divide the universal adhesive components into two bottles because the silane can be mixed with acidic monomers just before application ${ }^{14}$.

Another option of silane activation is heat treatment ${ }^{8}$ as it is described by the Arrhenius law that the hydrolysis reaction rate is temperature dependent ${ }^{14)}$. A previous study reported that the reaction rate of silane hydrolysis was more than 6 times higher when temperature was increased from $20^{\circ} \mathrm{C}$ to $50^{\circ} \mathrm{C}^{15)}$. In dentistry, warm airblowing of silane coupling agents was reported to enhance 
bonding to glass ceramics ${ }^{3,8)}$ and glass fiber posts ${ }^{16,17)}$. However, studies about effect of heat treatment on the bonding performance of silane-containing universal adhesives to glass ceramics are scarce. Additionally, warm air-blowing could also enhance the evaporation of water and volatile solvents from the adhesive after its application and hence prevent their negative effects on the bond strength and mechanical properties of the adhesive layer ${ }^{18)}$. Therefore, warm air-blowing might improve the adaptation and the bonding performance of silane-containing universal adhesives to glass ceramics, especially their bonding durability.

The purpose of this study was to evaluate the effect of warm air-blowing on the initial and long-term sealing ability of two one-bottle and one two-bottle silane-containing universal adhesives applied to lithium disilicate ceramics. In order to maximize stress to the interface, bonding to tapered cavities with a relatively high configuration factor (the ratio of bonded to unbonded surface area) was selected instead of flat surfaces. The analyses of adhesives' adaptation were performed using swept-source optical coherence tomography (SSOCT), a non-destructive imaging method which was proven capable of sensitively detecting gap formation at adhesive interfaces. The null hypotheses were (1) that there would be no significant difference in initial gap formation between the one-bottle and two-bottle silanecontaining universal adhesives, and (2) that warm air-blowing would not result in a significant difference in the sealing performance of the universal adhesives after thermocycling and a subsequent long-term water storage.

\section{MATERIALS AND METHODS}

Lithium disilicate glass ceramic blocks IPS e.max CAD (Ivoclar Vivadent, Schaan, Liechtenstein) with cavities prefabricated by the manufacturer were used in this study. The cavities were $3 \mathrm{~mm}$ deep and their shape was tapered (bottom diameter $2 \mathrm{~mm}$, upper diameter $4 \mathrm{~mm}$ ). The tested adhesives included one-bottle light-curing universal adhesives Scotchbond Universal (SBU; 3M, St. Paul, MN, USA) and Clearfil Universal Bond Quick (UBQ; Kuraray Noritake Dental, Tokyo, Japan), and the two-bottle self-curing universal adhesive Bondmer Lightless (BL; Tokuyama Dental, Tokyo, Japan). The flowable resin composite Estelite Flow Quick (Tokuyama Dental) was used to fill the cavities. The composition of these materials is presented in Table 1.

\section{Specimen preparation}

Prior to bonding, all blocks were cleaned with ethanol, ultrasonically cleaned in distilled water for $10 \mathrm{~min}$, air-dried, and randomly assigned to 3 groups $(n=10)$ according to the adhesive system used. The adhesives were applied with a disposable microbrush to the entire cavity surface and SBU was rubbed for $20 \mathrm{~s}$ according to the manufacturer's instructions. Then, the adhesives were air-blown either with normal air $\left(23 \pm 1^{\circ} \mathrm{C} ; \mathrm{N}, n=5\right)$ for $20 \mathrm{~s}$ using a dental air-syringe from a $10 \mathrm{~mm}$ distance, or with warm $\operatorname{air}\left(60 \pm 5^{\circ} \mathrm{C} ; \mathrm{W}, n=5\right)$ for $20 \mathrm{~s}$ using a heat blower (Dyson Supersonic Dryer, Dyson, Malmesbury, UK) from a $50 \mathrm{~mm}$ distance. The air temperature at the surface of the ceramic block was checked using a thermocouple and the distance of $50 \mathrm{~mm}$ was selected because the temperature near the heat blower's orifice was almost $100^{\circ} \mathrm{C}$ which could clinically cause burns

Table 1 The composition of materials used in this study

\begin{tabular}{|c|c|c|}
\hline Material (manufacturer) & Material type & Composition (batch number) \\
\hline $\begin{array}{l}\text { IPS e.max CAD } \\
\text { (Ivoclar Vivadent) }\end{array}$ & $\begin{array}{l}\text { Lithium disilicate } \\
\text { ceramic }\end{array}$ & $\mathrm{SiO}_{2}, \mathrm{Li}_{2} \mathrm{O}, \mathrm{K}_{2} \mathrm{O}, \mathrm{P}_{2} \mathrm{O}_{5}, \mathrm{ZrO}_{2}, \mathrm{ZnO}$, other oxides and ceramic pigments \\
\hline $\begin{array}{l}\text { Bondmer Lightless } \\
\text { (Tokuyama Dental) }\end{array}$ & $\begin{array}{l}\text { Two-bottle } \\
\text { universal adhesive }\end{array}$ & $\begin{array}{l}\text { Solution A: phosphoric acid monomer (3D-SR monomer), } \\
\text { MTU-6, HEMA, Bis-GMA, TEGDMA, acetone } \\
\text { Solution B: } \gamma \text {-MPTES, acryl borate catalyst, peroxide, acetone, } \\
\text { isopropyl alcohol, water ( } 019018)\end{array}$ \\
\hline $\begin{array}{l}\text { Scotchbond Universal } \\
(3 \mathrm{M})\end{array}$ & $\begin{array}{l}\text { One-bottle } \\
\text { universal adhesive }\end{array}$ & $\begin{array}{l}\text { Bis-GMA, 10-MDP, polyalkenoic acid Vitrebond copolymer, } \\
\text { HEMA, ethanol, water, filler, silane, initiators (669485) }\end{array}$ \\
\hline $\begin{array}{l}\text { Clearfil Universal Bond } \\
\text { Quick ER (Kuraray } \\
\text { Noritake Dental) }\end{array}$ & $\begin{array}{l}\text { One-bottle } \\
\text { universal adhesive }\end{array}$ & $\begin{array}{l}\text { 10-MDP, Bis-GMA, HEMA, hydrophilic aliphatic dimethacrylate, } \\
\text { colloidal silica, silane coupling agent, sodium fluoride, CQ, ethanol, } \\
\text { water (4C0008) }\end{array}$ \\
\hline $\begin{array}{l}\text { Estelite Flow Quick } \\
\text { (Tokuyama Dental) }\end{array}$ & $\begin{array}{l}\text { Flowable resin } \\
\text { composite }\end{array}$ & $\begin{array}{l}\text { Bis-MPEPP, TEGDMA, UDMA, silica-zirconia filler, silica-titania } \\
\text { fillers ( } 53 \% \text { filler by volume, } 0.04 \text { to } 0.6 \mu \mathrm{m} \text { particle size), CQ } \\
\text { (040026P) }\end{array}$ \\
\hline
\end{tabular}

MTU-6: 6-methacryloyloxyhexyl-2-thiouracil-5-carboxylate; HEMA: 2-hydroxyethyl methacrylate; Bis-GMA: bisphenol A-glycidyl methacrylate; TEGDMA: triethyleneglycol dimethacrylate; $\gamma$-MPTES: $\gamma$-methacryloyloxypropyltriethoxysilan e; 10-MDP: 10-methacryloyloxydecyl dihydrogen phosphate; CQ: camphorquinone; Bis-MPEPP: bisphenol A polyethoxy dimethacrylate; UDMA: urethane dimethacrylate. 
of soft tissues. After air-blowing, SBU and UBQ were light-cured for $10 \mathrm{~s}$ using a LED light-curing unit (1,470 $\mathrm{mW} / \mathrm{cm}^{2}$, Elipar DeepCure-L, 3M), whereas BL was not light-irradiated as recommended by the manufacturer. Then, the cavities were filled with the flowable resin composite in three 1-mm increments and each layer was light-cured for $20 \mathrm{~s}$. The specimens were stored in distilled water for $24 \mathrm{~h}$ at $37^{\circ} \mathrm{C}$ and then subjected to 10,000 thermocycles between $5^{\circ} \mathrm{C}$ and $55^{\circ} \mathrm{C}$ (dwell time $30 \mathrm{~s}$, transfer time $2 \mathrm{~s}$ ). Additionally, specimens which withstood thermocycling were stored in distilled water at $37^{\circ} \mathrm{C}$ for one year (the water was changed monthly).

\section{Investigation of gap formation using SS-OCT}

The SS-OCT observation of the filled cavities was performed immediately, after 24 -h water storage, 5,000 and 10,000 thermal cycles, and 1-year of water storage using IVS-2000 (Santec, Tokyo, Japan) with a laser light source of center wavelength $1,330 \mathrm{~nm}$ (bandwidth 110 $\mathrm{nm}$ ) and a sweep rate of $30 \mathrm{kHz}$. The laser beam scans the object in $\mathrm{X}$ and $\mathrm{Z}$ dimensions and the backscattered light detected by the system is digitized in time scale and subsequently analyzed in the Fourier domain to create a real-time cross-sectional 2-dimensional image of the specimen. The acquired images of $2,000 \times 1,000 \mathrm{px}$ resolution correspond to a $5 \times 5 \mathrm{~mm}(\mathrm{x}, \mathrm{z})$ scan range ${ }^{19)}$. Prior to the observation, the specimens were fixed on a metal stage using a tape to avoid unconventional surface reflections. The adaptation at cavity walls was observed at the angles of $0^{\circ}, 30^{\circ}, 60^{\circ}, 90^{\circ}, 120^{\circ}$, and $150^{\circ}$.

The measurement of gap length in the SS-OCT image was performed using the ImageJ software (version 1.48v, National Institutes of Health, Bethesda, Maryland, USA). After raw SS-OCT tomograms were imported to the software, a median filter was applied to decrease the background noise ${ }^{20)}$. An experimental threshold determination algorithm developed as a JAVA plugin for ImageJ was used for image analyses. The region of interest (ROI) was selected along the interface to highlight adaptation defects at cavity walls. The ratio of white pixels (gap) to the total length of the interface in ROI was automatically calculated by the plugin and expressed in percentages ${ }^{211}$.

\section{Statistical analysis}

Shapiro-Wilk test indicated that the gap length data were not normally distributed, so the non-parametric Kruskal Wallis test was used to compare the aging conditions within each tested group and followed by multiple comparisons using the Steel-Dwass method, $\alpha=0.05$ (JMP, version 13.2.1 for Windows, SAS Institute, Cary, NC, USA).

\section{RESULTS}

The results are presented in Table 2 and representative SS-OCT images for gap formation in Fig. 1. The gap formation of the normal air-blown groups $\mathrm{SBU}(\mathrm{N})$ and UBQ(N) were not significantly different from each other $(p=0.842)$ and exhibited significantly higher immediate gap formation than BL(N) $(p<0.001)$. After 24-h water storage, all the specimens of $U B Q(N)$ and $\mathrm{SBU}(\mathrm{N})$ completely detached from the cavities, so they were assigned a gap length of $100 \%$. In contrast, BL(N) presented no significant differences between immediate and 24 -h values $(p=1)$. After 5,000 thermal cycles, the gap formation of $\mathrm{BL}(\mathrm{N})$ increased significantly $(p<0.001)$ but remained similar after further thermocycling. After 1-year storage, four of five $\mathrm{BL}(\mathrm{N})$ specimens detached from the cavities, resulting in a significantly higher gap formation compared to that after 10,000 thermal cycles $(p=0.003)$.

In the warm air-blown groups, a significant reduction in immediate gap formation was observed for $\mathrm{SBU}(\mathrm{W})$ $(p<0.001)$, whereas $\mathrm{UBQ}(\mathrm{W})$ and $\mathrm{BL}(\mathrm{W})$ exhibited values similar to their respective normal air-blown groups $(p>0.05)$. The gap formation of BL(W) was significantly lower than those of SBU(W) and $\mathrm{UBQ}(\mathrm{W})(p<0.001)$. After $24 \mathrm{~h}$, all SBU(W) specimens were detached. The gap formation of UBQ(W) increased significantly after $24 \mathrm{~h}(p=0.011)$, but it was significantly lower compared to $\mathrm{UBQ}(\mathrm{N})(p<0.001)$. All UBQ(W) specimens detached from the cavities during the first day of thermocycling. Compared to the immediate gap formation, $\mathrm{BL}(\mathrm{W})$

Table 2 Results — relative gap length in percentages

\begin{tabular}{|c|c|c|c|c|c|}
\hline & Immediate & 24 hours & $5,000 \mathrm{TC}$ & $10,000 \mathrm{TC}$ & 1 year \\
\hline $\mathrm{BL}(\mathrm{N})$ & $10.9(0.8)^{\mathrm{aA}}$ & $11.5(0.9)^{\mathrm{aA}}$ & $48.0(1.4)^{\mathrm{aB}}$ & $49.2(2.3)^{\mathrm{aB}}$ & $92.3(15.7)^{\mathrm{ac}}$ \\
\hline $\mathrm{BL}(\mathrm{W})$ & $11.7(1.7)^{\mathrm{aA}}$ & $21.7(1.0)^{\mathrm{bB}}$ & $52.6(0.6)^{\mathrm{bC}}$ & $52.8(0.7)^{\mathrm{bC}}$ & $75.2(21.6)^{\mathrm{bL}}$ \\
\hline SBU (N) & $53.4(2.6)^{\mathrm{bA}}$ & $100 \quad(0.0)^{\mathrm{cB}}$ & $100 \quad(0.0)^{\mathrm{cB}}$ & $100 \quad(0.0)^{\mathrm{cB}}$ & $100 \quad(0.0)^{\mathrm{aB}}$ \\
\hline SBU (W) & $49.0(1.9)^{\mathrm{cA}}$ & $100 \quad(0.0)^{\mathrm{cB}}$ & $100 \quad(0.0)^{\mathrm{cB}}$ & $100 \quad(0.0)^{\mathrm{CB}}$ & $100 \quad(0.0)^{\mathrm{aB}}$ \\
\hline UBQ $(\mathrm{N})$ & $54.4(2.4)^{\mathrm{bA}}$ & $100 \quad(0.0)^{\mathrm{CB}}$ & $100 \quad(0.0)^{\mathrm{cB}}$ & $100 \quad(0.0)^{\mathrm{cB}}$ & $100 \quad(0.0)^{\mathrm{aB}}$ \\
\hline UBQ (W) & $53.3(2.7)^{\mathrm{bA}}$ & $55.8(0.0)^{\mathrm{dB}}$ & $100 \quad(0.0)^{\mathrm{cC}}$ & $100 \quad(0.0)^{\mathrm{cC}}$ & $100 \quad(0.0)^{\mathrm{aC}}$ \\
\hline
\end{tabular}

Different superscript letters indicate significant differences $(p<0.05)$ between groups; lowercase letters in columns and uppercase letters in rows.

BL: Bondmer Lightless; SBU: Scotchbond Universal; UBQ: Clearfil Universal Bond Quick ER; N: normal air; W: warm air; TC: thermal cycles. 


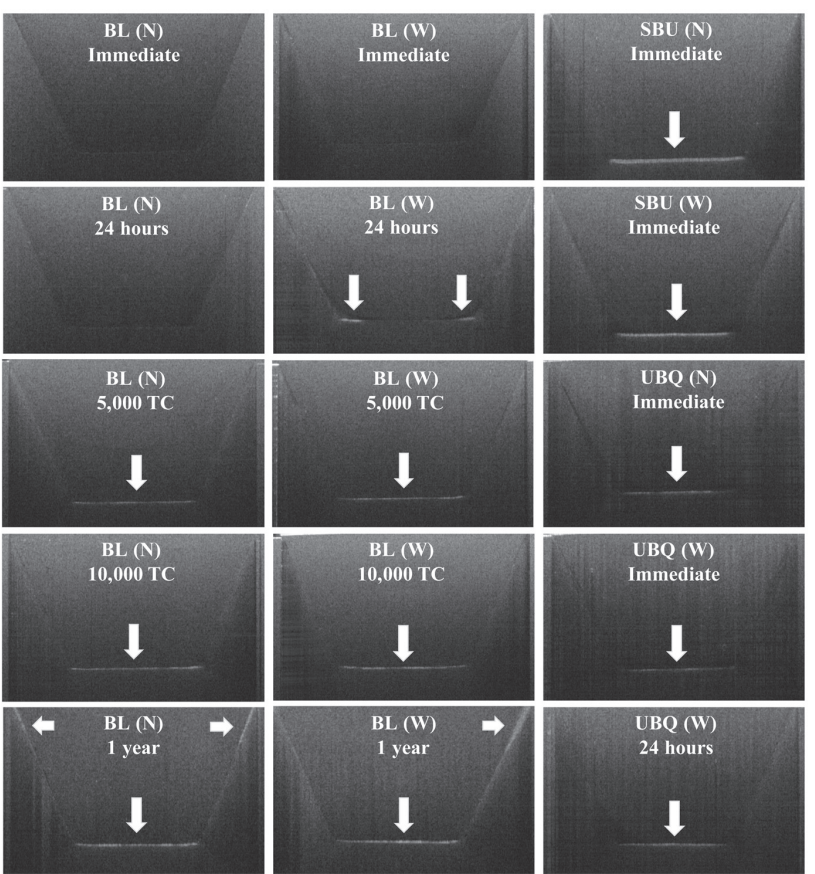

Fig. 1 Representative SS-OCT images of each observed group.

Gap formation was observed at the interfaces and is highlighted by the white arrows. A gradual increase in gap formation can be observed in the normal air-blown group of BL (first column) and in the warm air-blown group of BL (second column). In the third column, only immediate observations of SBU and UBQ are presented because the specimens detached from cavities within $24 \mathrm{~h}$ except for the warm air-blown group of UBQ.

BL: Bondmer Lightless; SBU: Scotchbond Universal; UBQ: Clearfil Universal Bond Quick ER; N: normal air; W: warm air; TC: thermal cycles

exhibited significantly higher 24 -h values $(p<0.001)$ and further increase was noted after 5,000 thermal cycles $(p<0.001)$. The gap formation of $\mathrm{BL}(\mathrm{N})$ was significantly lower than that of $\mathrm{BL}(\mathrm{W})$ after $24 \mathrm{~h}$ and thermocycling $(p<0.001)$. However, after 1-year water storage, BL(W) exhibited a significantly lower gap formation than BL(N) $(p=0.002)$ because only two BL(W) specimens detached from their cavities compared to four in $\mathrm{BL}(\mathrm{N})$ group.

\section{DISCUSSION}

The results of this study revealed that the gap formation of the two-bottle self-curing universal adhesive BL applied to lithium disilicate ceramics was significantly lower than those of the one-bottle light-curing universal adhesives SBU and UBQ, so the first null hypothesis had to be rejected. With normal air-blowing, SBU and UBQ exhibited a considerable immediate gap formation which increased dramatically during the 24-h water storage period, resulting in the detachment of all the resin composite specimens from their cavities. In the absence of hydrofluoric acid etching, these results indicate that $\gamma$-MPTS incorporated in SBU and UBQ was insufficient to achieve stable chemical bonding to lithium disilicate ceramics. This is in agreement with previous studies which have reported low bond strengths of one-bottle silane-containing universal adhesives to glass ceramics ${ }^{10,11)}$. The plausible reason is that $\gamma$-MPTS was pre-hydrolyzed in the acidic conditions of one-bottle universal adhesives and the silanol underwent a selfcondensation into inactive oligomers ${ }^{11,13)}$. On the other hand, the premature hydrolysis is prevented in the twobottle universal adhesive BL because the silane is mixed with the acidic monomers just before the application. The lower gap formation of BL observed in this study corroborates the previous finding that the addition of $\gamma$-MPTS to a one-bottle universal adhesive shortly preceding the application significantly improved the bond strength to glass ceramics ${ }^{11)}$. However, just oneday storage of the mixture reverted the positive effect ${ }^{11)}$ which demonstrates instability of the silane in the acidic conditions of universal adhesives.

Warm air-blowing of silane coupling agents was previously proven to improve the adhesion to glass ceramics ${ }^{3,8)}$ and glass fiber posts ${ }^{16,17)}$ due to the acceleration of the silane hydrolysis reaction ${ }^{14)}$. In this study, warm air $\left(60 \pm 5^{\circ} \mathrm{C}\right)$ was delivered using a heat blower to increase the temperature of silane-containing universal adhesives applied to lithium disilicate ceramics. Therefore, if any unhydrolyzed silane remained in the one-bottle universal adhesives, it could be activated by the warm air and hence improve the adhesion to lithium disilicate ceramics. The immediate SS-OCT observation in this study revealed that warm air-blowing significantly decreased the gap formation of SBU, but not that of UBQ. These results might indicate that some unhydrolyzed silane molecules were left in SBU, but hardly any in UBQ.

Besides the positive effect on the silane activation, warm air-blowing could also facilitate the solvent evaporation from the applied adhesives, thus improving the mechanical properties of the adhesive layer and enhancing the bonding performance ${ }^{22,23)}$. For SBU, all the resin-composite specimens dislodged from the cavities after 24-h water storage despite warm air-blowing. On the other hand, all warm air-blown UBQ specimens withstood 24-h water storage and only a slight increase in gap formation was noted. This different behavior can be explained by the different composition of these onebottle universal adhesives. SBU contains the hydrophilic monomer 2-hydroxyethyl methacrylate (HEMA) whose presence in a polymer chain increases its hydrophilicity and hence water uptake, resulting in reduced mechanical properties of HEMA-containing adhesives ${ }^{24,25)}$. Therefore, the infiltration of water into the adhesive layer of SBU could contribute to the dislodgement of the resin composite from cavities despite the slightly improved chemical interaction with the lithium disilicate achieved by warm air-blowing. To reduce the negative effects of HEMA, UBQ utilizes a multifunctional hydrophilic 
amide monomer, which contributes to a decrease in water sorption and an improvement in mechanical properties $^{26)}$. Combined with the positive effect of warm air-blowing on solvent evaporation, UBQ maintained its adaptation after the 24 -h water storage period due to the improved mechanical properties of the adhesive layer. This is in contrast to normal air-blowing which could not prevent the detachment of the resin composite specimens from the cavities after 24 -h water storage.

Compared to SBU and UBQ, the two-bottle selfcuring universal adhesive BL exhibited a significantly lower gap formation. The principal reason for this could be that the silane and acidic monomers are contained in separate bottles and they are mixed just before the application. Hence, the pre-hydrolysis and self-condensation of the silane in the bottle should be limited, and the silane should be able form a siloxane bond with the lithium disilicate surface, thus improving the bond strength and decreasing the gap formation. However, in contrast to the expected positive influence of warm-air blowing on the silane activation and the solvent evaporation, no improvement in the adaptation of BL was observed immediately after the resin composite placement and gap formation significantly increased after 24-h water storage compared to normal air-blowing. These results indicate that in the case of warm air-blowing, an adhesive layer was formed, whose mechanical properties were more likely to deteriorate with water sorption. In this study, warm air-blowing was performed from a $50 \mathrm{~mm}$ distance to control the temperature on the ceramic surface using a heat blower with an out-port of a diameter much larger than the orifice of cavity. This could lead to weaker air-stream compared with normal air-blowing from a $10 \mathrm{~mm}$ distance using a dental air-syringe, and hinder the optimal solvent evaporation from BL. Furthermore, increasing the temperature of the applied adhesive would also promote its chemical polymerization rate which could possibly entrap remaining solvents within the adhesive layer. As a consequence of the weaker air-stream and/or the faster chemical polymerization, warm air-blowing might produce a more porous adhesive layer and lead to the development of the adverse effect of water sorption on the mechanical properties and gap formation of BL.

The thermocycling test has often been used to investigate the durability of adhesive joints because it can inflict mechanical stress induced by utilizing the different thermal expansion coefficients of the bonded materials. Furthermore, changes in the temperature of water can also induce chemical stress and accelerate the bond hydrolysis. The magnitude of the mechanical stress at the interface is influenced by the configuration of the cavity prepared in the adherend material. When flat-to-flat surface adhesive specimens are subjected to thermocycling, the mechanical stress is relatively low and the degradation of the interface is mainly caused by hydrolysis. On the other hand, the tapered cavities (2 $\mathrm{mm}$ bottom diameter, $4 \mathrm{~mm}$ upper diameter, $3 \mathrm{~mm}$ depth) prepared in this study have a relatively high configuration factor which could amplify the mechanical stress to the interface resulting from the different thermal expansion of the resin composite and lithium disilicate ceramics. SBU specimens completely detached from the cavities prior to the start of thermocycling irrespective of the air-blowing technique along with the specimens in the normal air-blown UBQ group. Furthermore, the UBQ warm air-blown specimens which withstood the initial 24 -h water storage, detached soon after the start of thermal cycling. These results demonstrate that the stability of the bond between onebottle silane-containing universal adhesives and lithium disilicate ceramics is insufficient. On the other hand, all resin composite specimens bonded using BL survived thermocycling regardless of the air-blowing strategy, although the gap formation increased significantly.

An additional 1-year water storage was performed to investigate the hydrolytic stability of bonding to lithium disilicate ceramics ${ }^{27)}$ because there was no significant difference in the gap formation of BL between the 5,000 and 10,000 thermal cycles. In contrast to the results after 24-h water storage and thermocycling, the warm air-blown BL group exhibited a significantly lower gap formation than the normal air-blown group after 1-year water storage. With warm air-blowing, only two resin composite specimens failed, whereas four specimens dislodged from the cavities with normal air-blowing. These results indicate that warm air-blowing produced a more durable bond between BL and lithium disilicate ceramics than normal air-blowing, although the adhesive layer would have lower mechanical properties. Presumably, the heat delivered by warm air-blowing improved the chemical interaction of $\mathrm{BL}$ with lithium disilicate ceramics due to the increased activation of the silane. Nevertheless, the clinical applicability of heat blowers is limited due to the large nozzle diameter, so a warm-air-generating device connectable to a dental syringe should be developed.

\section{CONCLUSION}

Within the limitations of this study, it was concluded that the adaptation of one-bottle silane-containing universal adhesives to lithium disilicate ceramics without hydrofluoric acid etching was insufficient even in the short term. Warm air-blowing improved the sealing ability of one-bottle silane-containing universal adhesives only slightly, presumably due to the fact that most of the incorporated silane was pre-hydrolyzed during storage. On the other hand, a better adaptation to lithium disilicate ceramics was obtained with the two-bottle silane-containing universal adhesive. Warm air-blowing improved its sealing stability after 1 year of water storage; however, the gap formation was still considerable. Further research is required to evaluate the effect of warm air-blowing on the adaptation of universal adhesives to lithium disilicate ceramics when using a silane coupling agent in a separate application step. 


\section{ACKNOWLEDGMENTS}

This work was supported by the Ministry of Education, Culture, Sports, Science and Technology of Japan (Grant Numbers 18K09571 and 19K10106).

\section{DECLARATION OF INTEREST}

None.

\section{REFERENCES}

1) Attia A. Influence of surface treatment and cyclic loading on the durability of repaired all-ceramic crowns. J Appl Oral Sci 2010; 18: 194-200.

2) Kiomarsi N, Saburian P, Chiniforush N, Karazifard MJ, Hashemikamangar SS. Effect of thermocycling and surface treatment on repair bond strength of composite. J Clin Exp Dent 2017; 9: e945-e951.

3) Fabianelli A, Pollington S, Papacchini F, Goracci C, Cantoro A, Ferrari M, et al. The effect of different surface treatments on bond strength between leucite reinforced feldspathic ceramic and composite resin. J Dent 2010; 38: 39-43.

4) Ozcan M, Allahbeickaraghi A, Dündar M. Possible hazardous effects of hydrofluoric acid and recommendations for treatment approach: a review. Clin Oral Investig 2012; 16: $15-23$.

5) Meyer Filho A, Vieira LCC, Araújo É, Monteiro S. Effect of different ceramic surface treatments on resin microtensile bond strength. J Prosthodont 2004; 13: 28-35.

6) Della Bona A, Shen C, Anusavice KJ. Work of adhesion of resin on treated lithia disilicate-based ceramic. Dent Mater 2004; 20: 338-344.

7) Ahn D, Dhinojwala A. Silicone Surface Science. vol. 4. Dordrecht: Springer Netherlands; 2012. p. 23-58.

8) Shen C, Oh WS, Williams JR. Effect of post-silanization drying on the bond strength of composite to ceramic. J Prosthet Dent 2004; 91: 453-458.

9) Mamanee T, Takahashi M, Nakajima M, Foxton RM, Tagami J. Initial and long-term bond strengths of one-step self-etch adhesives with silane coupling agent to enamel-dentincomposite in combined situation. Dent Mater J 2015; 34: 663670.

10) Noda $Y$, Nakajima M, Takahashi M, Mamanee T, Hosaka K, Takagaki T, et al. The effect of five kinds of surface treatment agents on the bond strength to various ceramics with thermocycle aging. Dent Mater J 2017; 36: 755-761.

11) Yoshihara K, Nagaoka N, Sonoda A, Maruo Y, Makita Y, Okihara T, et al. Effectiveness and stability of silane coupling agent incorporated in "universal" adhesives. Dent Mater 2016; 32: 1218-1225.

12) Lung CYK, Matinlinna JP. Aspects of silane coupling agents and surface conditioning in dentistry: an overview. Dent Mater 2012; 28: 467-477.
13) Chen B, Lu Z, Meng H, Chen Y, Yang L, Zhang H, et al. Effectiveness of pre-silanization in improving bond performance of universal adhesives or self-adhesive resin cements to silica-based ceramics: Chemical and in vitro evidences. Dent Mater 2019; 35: 543-553.

14) Matinlinna JP, Lung CYK, Tsoi JKH. Silane adhesion mechanism in dental applications and surface treatments: A review. Dent Mater 2018; 34: 13-28.

15) Jiang H, Zheng Z, Li Z, Wang X. Effects of Temperature and Solvent on the Hydrolysis of Alkoxysilane under Alkaline Conditions. Ind Eng Chem Res 2006; 45: 8617-8622.

16) Monticelli F, Toledano M, Osorio R, Ferrari M. Effect of temperature on the silane coupling agents when bonding core resin to quartz fiber posts. Dent Mater 2006; 22: 1024-1028.

17) Samimi P, Mortazavi V, Salamat F. Effects of heat treating silane and different etching techniques on glass fiber post push-out bond strength. Oper Dent 2014; 39: e217-e224.

18) Manuja N, Nagpal R, Pandit I. Dental adhesion: mechanism, techniques and durability. J Clin Pediatr Dent 2015; 36: 223234.

19) Hayashi J, Shimada Y, Tagami J, Sumi Y, Sadr A. Realtime imaging of gap progress during and after composite polymerization. J Dent Res 2017; 96: 992-998.

20) Bakhsh TA, Sadr A, Shimada Y, Tagami J, Sumi Y. Noninvasive quantification of resin-dentin interfacial gaps using optical coherence tomography: Validation against confocal microscopy. Dent Mater 2011; 27: 915-925.

21) Bista B, Sadr A, Nazari A, Shimada Y, Sumi Y, Tagami J. Nondestructive assessment of current one-step self-etch dental adhesives using optical coherence tomography. J Biomed Opt 2013; 18: 76020.

22) Klein-Júnior CA, Zander-Grande C, Amaral R, Stanislawczuk R, Garcia EJ, Baumhardt-Neto R, et al. Evaporating solvents with a warm air-stream: effects on adhesive layer properties and resin-dentin bond strengths. J Dent 2008; 36: 618-625.

23) Yonekura K, Hosaka K, Tichy A, Taguchi K, Ikeda M, Thanatvarakorn O, et al. Air-blowing strategies for improving the microtensile bond strength of one-step self-etch adhesives to root canal dentin. Dent Mater J 2020; 39: 892-899.

24) Hosaka K, Nakajima M, Takahashi M, Itoh S, Ikeda M, Tagami J, et al. Relationship between mechanical properties of one-step self-etch adhesives and water sorption. Dent Mater 2010; 26: 360-367.

25) Takahashi M, Nakajima M, Hosaka K, Ikeda M, Foxton RM, Tagami J. Long-term evaluation of water sorption and ultimate tensile strength of HEMA-containing/-free one-step self-etch adhesives. J Dent 2011; 39: 506-512.

26) Kuno Y, Hosaka K, Nakajima M, Ikeda M, Klein Junior CA, Foxton RM, et al. Incorporation of a hydrophilic amide monomer into a one-step self-etch adhesive to increase dentin bond strength: Effect of application time. Dent Mater J 2019; 38: 892-899.

27) Foxton RM. Adhesion of dual-cure resin cement to ceramic: Action of ceramic primers. Fixed Prosthodontics Department of Restorative Sciences Faculty of Dentistry Tokyo Medical and Dental University; 2002. p. 20-48. 